\title{
On the Location of Zeros of Some Polynomials
}

\author{
Dragomir M. Simeunović
}

\begin{abstract}
In this paper we determine the regions in the complex plane containing zeros of some polynomials.
\end{abstract}

In this paper we consider the polynomial

$$
P(z)=z^{n}+a_{p} z^{n-p}+a_{p+1} z^{n-p-1}+\cdots+a_{n}, a_{p} \neq 0, p<n .
$$

The location of zeros of the polynomials in the complex plane, depending on its coefficients was studied by many authors. Here we cite a result obtained by P. Montel [1] and a result by H. Guggenheimer [2] which are, respectively, as follows:

$\left(R_{1}\right)$ : All the zeros of the polynomial (1) are in the region

$$
|z|<2 \max \left|a_{k}\right|^{\frac{1}{k}}, p \leq k \leq n .
$$

$\left(R_{2}\right)$ : All the zeros of the polynomial (1) are in the region

$$
|z|<r
$$

where $r>1$ is the root of the equation

$$
r^{p}-r^{p-1}-\left|a_{q}\right|=0
$$

and where

$$
\left|a_{q}\right|=\max \left|a_{k}\right|, p \leq k \leq n .
$$

In this paper we prove the following theorem.

Theorem 1. Let $c_{k}, p \leq k \leq n$ be the positive parameters, where

$$
A_{c}=\max \left(\frac{\left|a_{k}\right|}{c_{k}}\right), p \leq k \leq n,
$$

and

$$
M_{c}=\max \left(c_{k}\right)^{\frac{1}{k}}, p \leq k \leq n .
$$

Then all zeros of the polynomial (1) are in the region

$$
|z|<r_{c} M_{c} \text {, }
$$

2010 Mathematics Subject Classification. Primary: 12D10.

Key words and phrases. Zeros of the polynomials, region of zeros. 
where $r_{c}>1$ is the root of the equation

$$
r^{p}-r^{p-1}-A_{c}=0 .
$$

Proof of Theorem 1. From (7) we have

$$
c_{k}{ }^{\frac{1}{k}} \leq M_{c}
$$

that is

$$
c_{k} \leq M_{c}^{k}, p \leq k \leq n .
$$

From (1), for $|z|>M_{c}$, having (6) and (10) in mind, we obtain

$$
\begin{aligned}
|P(z)| & =|z|^{n}\left(1-\left(\frac{\left|a_{p}\right|}{|z|^{p}}+\frac{\left|a_{p+1}\right|}{|z|^{p+1}}+\cdots+\frac{\left|a_{n}\right|}{|z|^{n}}\right)\right) \\
& =|z|^{n}\left(1-\left(\frac{\left|a_{p}\right|}{c_{p}} \cdot \frac{c_{p}}{|z|^{p}}+\frac{\left|a_{p+1}\right|}{c_{p+1}} \cdot \frac{c_{p+1}}{|z|^{p+1}}+\cdots+\frac{\left|a_{n}\right|}{c_{n}} \cdot \frac{c_{n}}{|z|^{n}}\right)\right) \\
& \geq|z|^{n}\left(1-A_{c}\left(\frac{c_{p}}{|z|^{p}}+\frac{c_{p+1}}{|z|^{p+1}}+\cdots+\frac{c_{n}}{|z|^{n}}\right)\right) \\
& >|z|^{n}\left(1-A_{c}\left(\frac{M_{c}^{p}}{|z|^{p}}+\frac{M_{c}^{p+1}}{|z|^{p+1}}+\cdots+\frac{M_{c}^{n}}{|z|^{n}}+\cdots\right)\right) \\
& =|z|^{n}\left(1-\frac{A_{c} M_{c}^{p}}{|z|^{p}}\left(1+\frac{M_{c}}{|z|}+\left(\frac{M_{c}}{|z|}\right)^{2}+\cdots+\left(\frac{M_{c}}{|z|}\right)^{n-p}+\cdots\right)\right) \\
& =|z|^{n}\left(1-\frac{A_{c} M_{c}^{p}}{|z|^{p}-|z|^{p-1} M_{c}}\right),
\end{aligned}
$$

that is

$$
|P(z)|>|z|^{n}\left(1-\frac{A_{c} M_{c}^{p}}{|z|^{p}-|z|^{p-1} M_{c}}\right) .
$$

For

$$
|z| \geq r_{c} M_{c}
$$

from (11) we have $|P(z)|>0$, that is $|P(z)| \neq 0$.

This means that all zeros of the polynomial (1) are in the region (8).

Taking different positive values for parameters $c_{k}$, we obtain several particular results from Theorem 1 .

For

$$
c_{k}=\frac{\left|a_{k}\right|}{2^{p-1}}, p \leq k \leq n,
$$

the following result from Theorem 1 is obtained:

$\left(R_{3}\right)$ : All zeros of the polynomial (1) are in the region

$$
|z|<2 \max \left(\frac{\left|a_{k}\right|}{2^{p-1}}\right)^{\frac{1}{k}}, p \leq k \leq n .
$$


Proof of $\left(R_{3}\right)$. Having (13) in mind, from (6) and (7) we obtain

$$
A_{c}=2^{p-1}
$$

and

$$
M_{c}=\max \left(\frac{\left|a_{k}\right|}{2^{p-1}}\right)^{\frac{1}{k}}, p \leq k \leq n .
$$

In this case the equation (9) reduces to equation

$$
r^{p}-r^{p-1}-2^{p-1}=0,
$$

whose positive root is

$$
r_{s}=2
$$

and region (8) reduces to region (14).

The region (14) for $p \geq 2$ is smaller than the region (2). For $p=1$ the region (14) reduces to the region (2).

We demonstrate the other case by giving an example.

Example 1. The zeros of the polynomial

$$
P(z)=z^{5}-8 z^{2}+11 z+20,
$$

where $p=3$, according to result $\left(R_{2}\right)$ are in the region

$$
|z|<3.1 \text {, }
$$

where $r>1$ is the root of the equation

$$
r^{3}-r^{2}-20=0, \quad(3<r<3.1),
$$

and from (14) follows that all zeros of the polynomial (19) are in the region

$$
|z|<2.76 \text {. }
$$

\section{REFERENCES}

[1] P. Montel, Sur quelques limites pour les modules des zéros des polynomes, Comment. Math. Helv., Vol. 7 (1934-35), 178-200.

[2] H. Guggenheimer, On a note by Q.G. Mohammad, Amer. Math. Monthly, 71 (1964), 54-55.

[3] M. Marden, Geometry of Polynomials, Amer. Math. Soc. Providence, RI. 2005.

Dragomir M. Simeunović Mike Alasa 8 11000 BELGRADE Serbia 